\title{
Follow the glow
}

\author{
Sunil Singhal, MD
}

\author{
From the Department of Thoracic Surgery, University of Pennsylvania School of Medicine, Philadelphia, Pa. \\ Disclosures: Author has nothing to disclose with regard to commercial support. \\ Received for publication April 24, 2017; accepted for publication May 23, 2017; available ahead of print June 17, \\ 2017. \\ Address for reprints: Sunil Singhal, MD, Department of Thoracic Surgery, University of Pennsylvania School of \\ Medicine, 3400 Spruce St, 6 Silverstein Pavilion, Philadelphia, PA 19104 (E-mail: sunil.singhal@uphs.upenn. \\ edu). \\ J Thorac Cardiovasc Surg 2017; 154:1119-20 \\ $0022-5223 / \$ 36.00$ \\ Copyright (C) 2017 by The American Association for Thoracic Surgery \\ http://dx.doi.org/10.1016/j.jtcvs.2017.05.078
}

In a recent article, Hackey and colleagues ${ }^{1}$ report on a feasibility study to determine whether preoperative tumor tattooing by navigational bronchoscopy can be used to localize tumors and perform sentinel lymph node (SLN) staging in patients with suspected early stage lung cancer. Although dye localization and SLN mapping have been studied in the past, this study is unique in that the investigators used a near-infrared (NIR) contrast dye that provides a fluorescent glow to the tumor to do the tumor tattooing. By passive drainage, the NIR dye is then localized to the SLN.

Small pulmonary nodules continue to be a surgical challenge. These lesions can be difficult to find intraoperatively, particularly if they are deep to the pleural surface or they are small and hard to palpate. To solve this problem, investigators have tried a slew of localization techniques, including percutaneous wire placement, injection of dyes, intraoperative imaging with ultrasound, fluoroscopy, and molecular imaging devices. ${ }^{2}$

Many types of injectable dyes have been used, including methylene blue, lioplo, cyanoacrylate, India ink, and technetium-99m dye. There are several limitations to these techniques that include dye diffusion between time of injection and surgery, difficulty in dye visualization during operation, limited information on lesion depth, and potential risk of embolism and cerebrovascular accident. In this study, none of these complications arose, and the use of the NIR dye showed high sensitivity.

These dyes are typically injected by 1 of 2 methods: guidance by computed tomography or navigational bronchoscopy. Over the past 5 years, several series have shown that navigational bronchoscopy has advantages over transthoracic approaches, such as minimal exposure to radiation and the ability to accomplish the marking in a single setting in the operating room. This avoids complicated coordination between other departments such as interventional radiology, which can be disruptive to the surgical flow. Also, the pneumothorax rates with percutaneous techniques for nodule identification are significantly reduced. Recent data suggest that there is less diffusion of the dye than typically seen with percutaneous approaches.

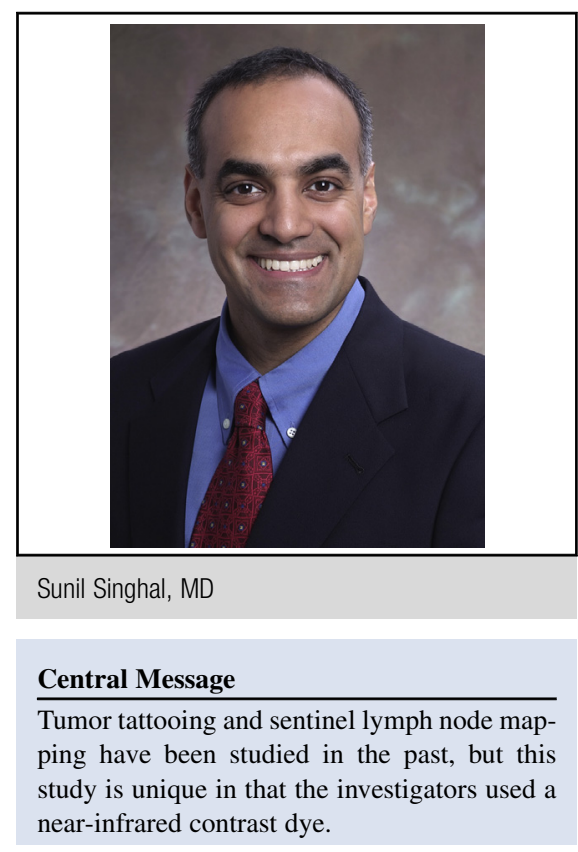

See Article page 1110 .

An added feature of this study by Hackey and colleagues ${ }^{1}$ was using drainage of the dye from the tumor as a method for SLN mapping. In several studies, intraoperative SLN mapping using various tracers, such as methylene blue, isosulfan blue, fluorescein, technetium-99m, and indocyanine green, have been used. ${ }^{3}$ In most of those cases, the dye was injected directly into the tumor. In the largest multiinstitutional report to date, ${ }^{4} 43$ patients had cancer and underwent an attempted complete resection, with 39 undergoing SLN mapping. One or more SLNs were identified in 24 of those 39 patients $(61.5 \%)$, with accurate indication (ie, no other nodes positive for cancer if SLN negative) in 20 patients $(83.3 \%)$. Overall, the SLN mapping procedure was found to be accurate in 20 of 39 patients $(51.2 \%)$. The trial was terminated early based on disappointing accuracy.

The article by Hackey and colleagues ${ }^{1}$ is a nice follow-up on earlier work on tumor tattooing using NIR dye by transthoracic injection. In that study, the tumor tattooing was performed using navigational bronchoscopy. Specifically, 12 patients with clinical T1 N0 non-small cell lung cancers who were scheduled for video-assisted thoracoscopic surgery sublobar resection underwent intraoperative navigational bronchoscopy. Once the nodules were located, they were injected with up to $2.5 \mathrm{mg}$ NIR dye (indocyanine green). Following peritumoral injection, the patients were repositioned in a lateral decubitus position and underwent 
video-assisted thoracoscopic surgery. In all 12 patients, the peritumoral tattoo was visualized through to the pleural surface. Ten patients ultimately had lung cancer. The advantage of the NIR dyes is deeper visualization into the lung compared with dyes that are visible in the 400 to $700 \mathrm{~nm}$ optical range. After the wedge of the nodules, patients underwent removal of the SLN that was fluorescent due to the drainage of the NIR dye from the tumor.

There are several nice findings the study by Hackey and colleagues. ${ }^{1}$ The use of NIR dye allowed for a deeper penetration with the endoscope compared with India ink. The entire procedure only added 30 minutes to each case and the SLN was defined as the first draining lymph node that was fluorescent. Another finding was that the drainage of the dye from the tumor to the first lymph node occurred rapidly; that is, during the time it took to position the patient and start the procedure. In 8 out of 12 patients, the first draining SLN was found in the mediastinum, not the hilum. It would be interesting to know whether the 4 patients who underwent cervical mediastinoscopy had any changes to their lymphatic drainage pattern after the natural tissue planes were disrupted.
The oncologic benefits of SLN mapping are still unclear, but if it does prove to be important, NIR dyes clearly will have an important role. In this pilot study by Hackey and colleagues, ${ }^{1}$ only 1 patient had a positive lymph node, so it is difficult to draw broad conclusions about the influence of SLN mapping. However, as stated by the authors, this was a feasibility study and that goal was accomplished.

Moving forward, we excitedly await the results of a larger study to validate these results and to learn more about efforts to bring this innovation to the thoracic surgery community to help solve a vexing problem.

\section{References}

1. Hachey KJ, Digesu CS, Armstrong KW, Gilmore DM, Khullar OV, Whang B, et al. A novel technique for tumor localization and targeted lymphaticmapping in earlystage lung cancer. J Thorac Cardiovasc Surg. 2017;154:1110-8.

2. Keating J, Singhal S. Novel methods of intraoperative localization and margin assessment of pulmonary nodules. Semin Thorac Cardiovasc Surg. 2016;28: 127-36.

3. Miyoshi S. Intraoperative nodal staging: role of sentinel node technology. Thorac Surg Clin. 2013;23:357-68.

4. Liptay MJ, D’Amico TA, Nwogu C, Demmy TL, Wang XF, Gu L, et al. Intraoperative sentinel node mapping with technitium-99 in lung cancer: results of CALGB 140203 multicenter phase II trial. J Thorac Oncol. 2009;4:198-202. 